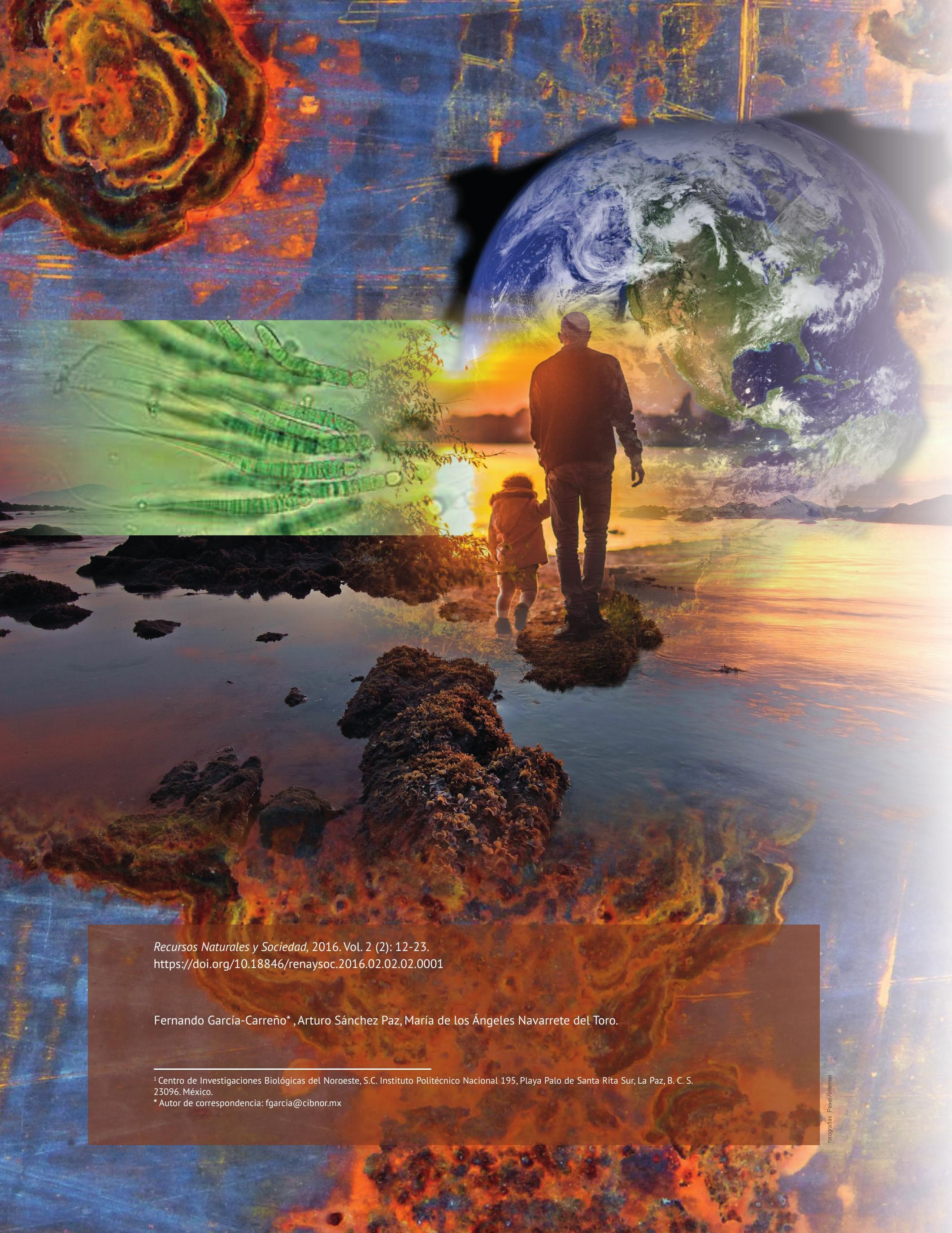




\section{La oxidación dualidad vida y muerte Oxidation, life and death duality}

\section{Resumen}

La oxidación es un proceso fisicoquímico que ocurre de forma natural tanto en la materia inorgánica como en los seres vivos; la principal forma de oxidación en seres vivos se debe al oxígeno. Oxidación deriva su nombre de "Oxígeno", $\mathrm{O}_{2}$, el gas que compone el $21 \%$ de la atmósfera; concentración que ha variado a lo largo de la historia de la tierra. El oxígeno es un elemento muy reactivo, por lo que en planetas sin vida solo se encuentra formando compuestos con otros elementos, como el agua, $\mathrm{H}_{2} \mathrm{O}$, o carbonatos, $\mathrm{CaCO}_{3}$

Los primeros seres vivos, los procariontes, fueron unicelulares, simples y anaerobios y obtenían energía por medio de oxidación por otros compuestos. No fue hasta que otros organismos adquirieron la habilidad de fotosintetizar que apareció el oxígeno molecular en la atmósfera, lo que causó el surgimiento de organismos aerobios.

El oxígeno promovió la gran diversidad de seres que ahora habitan el planeta. A través de la oxidación de los nutrientes se obtiene la energía necesaria para que los organismos mantengan los procesos celulares. El proceso es aproximadamente $98 \%$ eficiente, el $2 \%$ restante genera compuestos que son más oxidantes y que pueden dañar componentes celulares. Durante el proceso evolutivo se han seleccionado diversos mecanismos antioxidantes cuya función es prevenir la oxidación dañina. La hipótesis del estrés oxidativo ha fallado en explicar por qué los atletas que consumen más oxígeno viven más, y por qué la expectativa de vida del humano durante el Siglo XX aumentó 30 años. Aun así, y sin sustento científico se ha promovido el consumo masivo de "antioxidantes" para mantener la salud y vivir más. Este ensayo tiene como objeto exponer un análisis 
del importante impacto que el oxígeno ha tenido en la vida de nuestro planeta.

Palabras clave: Oxígeno, estrés oxidativo, oxidantes, evolución.

\section{Abstract}

Oxidation, a natural physicochemical process originating energy, which affects both inorganic matter and living beings. Oxidation derives from "Oxygen" $\left(\mathrm{O}_{2}\right)$, which amounts for $21 \%$ of the atmosphere gases; concentration that has changed throughout Earth's history. Oxygen reacts easily that is why it occurs only forming compounds as water $\left(\mathrm{H}_{2} \mathrm{O}\right)$ or carbonates $\left(\mathrm{CaCO}_{3}\right)$ in lifeless planets. First living beings, prokaryotes, were simple unicellular anaerobes that obtained energy using other oxidants. It was until photosynthetic organisms appeared on the primitive Earth that molecular oxygen accumulated in the atmosphere causing the proliferation of aerobe organisms. Oxygen is the reason behind the huge diversity of living things on the planet. By oxidizing food nutrients, organisms obtain energy for cellular processes. The process is $98 \%$ efficient; the other $2 \%$ yield more oxidative compounds with the capability of cell damage; antioxidant mechanisms have evolved in aerobes to prevent it. The oxidative stress hypothesis has failed to explain why athletes that breathe more oxygen live longer and why life expectancy for humans has increased 30 years in the last century. Even so and with no scientific evidence, the ingestion of massive amounts of antioxidants to promote health and live longer have been endorsed. This essay deals with the importance of oxygen promoting biodiversity on planet Earth.

Key words: Oxygen, oxidative stress, oxidants, evolution

\section{Introducción}

¿Te has preguntado por qué una manzana mordida se obscurece? 0 ¿por qué un clavo se oxida al aire libre? El culpable de esto es el oxígeno. Si bien nuestra atmósfera tiene una composición en la que el oxígeno sólo participa con el $21 \%$ del total de gases (el nitrógeno es el gas más abundante, con el 78\%), este elemento, debido a algunas características fisicoquímicas que describiremos más adelante, es muy reactivo. Quizás no lo sepas, pero las primeras formas vivientes en la tierra liberaban oxígeno molecular $\left(\mathrm{O}_{2}\right)$ como producto de desecho, y este gas contribuyó a aumentar la complejidad y diversidad biológica de nuestro planeta.

\section{¿Qué es la oxidación?}

La oxidación es un proceso fisicoquímico que ocurre de forma natural tanto en la materia inorgánica como en los seres vivos. La oxidación transforma elementos y compuestos químicos y del proceso se obtiene energía. A pesar de nuestra incapacidad para ver los átomos oxidándose, hemos sido testigos del proceso de la oxidación: un pedazo de hierro, comúnmente llamado "fierro," reacciona con la humedad y el oxígeno del aire,yprogresivamente se puede observar la formación de 
una delgada capa de óxido de hierro $\left(\mathrm{Fe}_{2} \mathrm{O}_{3} \cdot \mathrm{H}_{2} \mathrm{O}\right)$, o herrumbre rojizo (Fig. 1). Este proceso también se llama corrosión electroquímica. Este es un ejemplo muy obvio, pero no el único. La oxidación también ocurre cuando el gas de la cocina, o un pedazo de madera, ambos contienen carbono, reaccionan con el oxígeno del aire y producen dióxido de

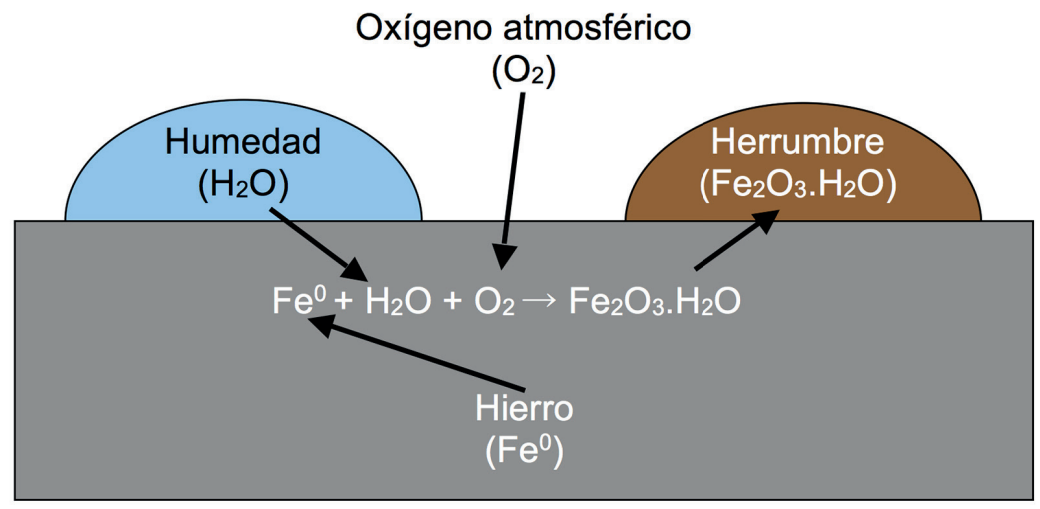

Figura 1. Representación esquemática de la reacción de oxidación del hierro.

carbono $\left(\mathrm{CO}_{2}\right)$. En estos ejemplos se demuestra que la combustión libera energía en forma de calor. Otro ejemplo es la oxidación de los alimentos que ingerimos para obtener la energía que nos permite realizar nuestras actividades vitales cotidianas. Los organismos respiradores, el término es aerobios, como el humano, oxidamos los nutrientes de los alimentos que hemos ingerido por estar hechos de carbono y como producto de desecho generamos bióxico de carbono, $\mathrm{CO}_{2}$, si, uno de los "gases invernadero," que eliminamos al expirar, expeler el aire de los pulmones. El proceso ocurre en las células por una serie de reacciones químicas que llevan al oxígeno a formar agua, $\mathrm{H}_{2} \mathrm{O}$. El obscurecimiento del plátano, la manzana y el aguacate son resultado de oxidación.

\section{Oxígeno}

El actor principal de esta historia es el oxígeno. Este elemento fue nombrado así por Antonio Lavoisier en 1777; debido a que creyó que el oxígeno formaba parte de todos los ácidos [oxígeno: del griego ógúc (oxys)

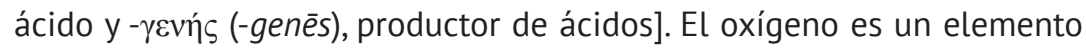
químico biatómico, $\mathrm{O}_{2}$, (2 átomos de oxígeno forman una molécula) y por ello se le llama oxígeno molecular. El $\mathrm{O}_{2}$ es la forma alótropica ${ }^{1}$ de oxígeno más abundante en la atmósfera. Otros alótropos son el ozono, $\mathrm{O}_{3}$, que se forma en la zona superior de la atmósfera y por la combustión de los motores de combustión interna. El ozono es más reactivo que el oxígeno molecular, más oxidante. Un alótropo recién descubierto es la molécula metaestable ${ }^{2}$ de tetraoxígeno, $\mathrm{O}_{4}$, la que es más oxidante de los alótropos del oxígeno. Esta forma alotrópica es tan oxidante que se ha pensado utilizarla como comburente en naves espaciales. Hay que recordar que fuera de la atmósfera no hay oxígeno y por lo tanto las naves espaciales deben llevar su propio oxígeno, así como también los tripulantes.

Como todo elemento químico, el oxígeno se encuentra clasificado en la tabla periódica dentro de una familia con 6 electrones en el nivel energético externo. Como el máximo posible de electrones en

\footnotetext{
${ }^{1}$ En química, se denomina alotropía a la propiedad que poseen algunos elementos químicos, como el oxígeno, de poseer estructuras moleculares diferentes.

${ }^{2}$ Metaestable, que puede cambiar a un estado más estable.
} 
ese nivel es 8 , el oxígeno puede tomar hasta 2 electrones de otros átomos. Por ello cuando reacciona con el hidrógeno, que sólo tiene un electrón, requiere dos átomos de hidrógeno para producir agua $\left(\mathrm{H}_{2} \mathrm{O}\right)$. Esta caracterísica hace que el oxígeno sea muy ávido para capturarlos, y técnicamente se le llama "electronegativo"; siendo el segundo elemento más electronegativo después del flúor, y es su electronegatividad lo que le confiere su poder oxidante $\tan$ reactivo. Es tan reactivo que en los planetas no habitados no existe como oxígeno molecular. Se encuentra formando compuestos como el óxido de hierro $(\mathrm{FeO})$ que da su color al planeta rojo.

En la tierra se encuentra como oxígeno molecular debido a que hay organismos fotosintéticos oxigénicos, tales como las plantas y cianobacterias, que lo producen como desecho de la fotosíntesis.

Por ello, los astrobiólogos (científicos que buscan señales de vida en otros planetas) buscan evidencias de vida como la presencia de oxígeno molecular. El oxígeno es tan electronegativo que los organismos respiradores lo usan como aceptor final de electrones, reacción que genera energía libre que es usada para las funciones de las células.

\section{Potencial Redox}

$\mathrm{Si}$ bien el oxígeno tiene la capacidad de "capturar" electrones, otros elementos son conocidos por su capacidad de cederlos.

Un elemento reductor, como el hidrógeno, es aquel que tiende a ceder electrones. El "potencial redox" es una forma de medir la energía de oxidación (potencial de oxidación) y/o reducción (potencial de reducción) de un par de sustancias, una que oxida y otra que reduce. El "potencial redox" se mide en voltios, Eh.

Un valor positivo indica que la reacción de oxidación ocurrirá espontáneamente, es decir, no requiere aporte de energía del medio. Un valor negativo indica que para que la reacción ocurra se requiere energía del medio. En la célula, la oxidación de glucosa, un azúcar del alimento, ocurre usando oxígeno como aceptor final de electrones. Por el contrario, la síntesis de glucosa, un proceso de reducción del carbono durante la fotosíntesis requiere energía, la energía luminosa del sol.

\section{Vida y el "planeta Oxígeno"}

Dos condiciones sine qua non ${ }^{3}$ determinaron la aparición de organismos aerobios ${ }^{4}$ en el planeta: 1) La concentración del oxígeno molecular en la atmósfera, y 2) la solubilidad de este en agua. Además de sales minerales y otros compuestos, el agua contiene gases. Entre ellos el oxígeno. Debido a su coeficiente de solubilidad, el oxígeno se encuentra en una concentración adecuada en el agua, al menos en la superficie de lagos y mares.

Esta característica permite que haya organismos acuáticos que respiran a través de branquias como peces, camarones y pulpos. La solubilidad del oxígeno molecular en agua depende de la temperatura y obedece a la Ley de Henry: "a mayor temperatura

${ }^{3}$ sine qua non, significa condición indispensable para que algo ocurra.

${ }^{4}$ Organismos que respiran, requieren utilizar oxígeno como aceptor terminal de electrones que se obtienen compuestos del alimento. respiración en ciencia se entiende a la capacidad de usar oxígeno para obtención de energía a partir de compuestos carbonados obtenidos en el alimento. Los organismos acuáticos usan branquias para obtener el oxígeno disuelto en el agua, así como los organismos terrestres usan pulmones para obtener oxígeno del aire. 
menos oxígeno disuelto" lo que tiene consecuencias importantes para la vida en los mares. A $25{ }^{\circ} \mathrm{C}$ y una atmósfera de presión, como la que se da a nivel del mar, el agua dulce contiene 6 mililitros $(\mathrm{mL})$ de oxígeno por litro de agua y el agua de mar $5 \mathrm{~mL}$. A $5{ }^{\circ} \mathrm{C}$ la concentración aumenta a 9 $\mathrm{mL}$ por $\mathrm{L}$ de agua dulce y a 7 de agua de mar, $50 \%$ más que a $25^{\circ} \mathrm{C}$. Por ello, el aumento de la temperatura de la atmósfera, como el que ocurre debido al calentamiento global, tendrá consecuencias, ya que, además de las causadas por el aumento de la concentración de $\mathrm{CO}_{2}$ en la atmósfera, la concentración de oxígeno en los océanos podrá no ser la necesaria para muchas especies que habitan los mares.

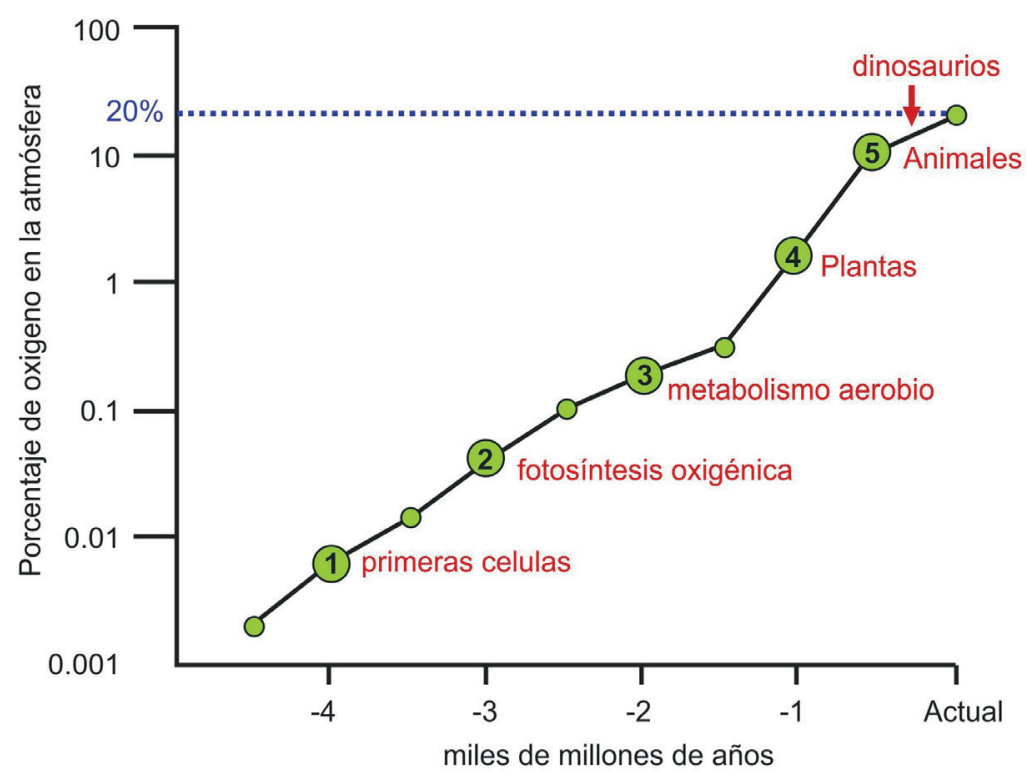

Figura 2. Aumento progresivo en la concentración de oxígeno molecular en la atmósfera terrestre

El oxígeno es el elemento más abundante, por masa, en la tierra y el tercero en el universo después de hidrógeno y helio. Casi el $50 \%$ de la masa de la tierra es oxígeno. Este dato no es sorprendente si sabemos que el agua está formada por la reacción entre hidrógeno y oxígeno, también forma carbonatos como el encontrado en la calcita $\left(\mathrm{CaCO}_{3}\right)$, mineral que contribuye con $4 \%$ a la masa de la tierra. Como ya se mencionó, el oxígeno es tan reactivo que en la tierra primigenia sólo se encontraba formando compuestos, como el $\mathrm{CO}_{2}$. Nuestro planea recibió el nombre de "Tierra" por que para quienes la nombraron lo primordial era la parte continental.
Cuando se supo que los océanos cubren el $70 \%$ de la superficie terrestre se propuso llamarla "Oceanía”. Ahora que sabemos que el principal componente del planeta es oxígeno, no es descabellado querer bautizarlo como "Oxígeno", como ya alguien ha propuesto Ilamarle "Vida" por esta característica sine qua non.

Los organismos fotosintéticos no fueron los primeros seres vivos.La vida inició en ausencia de oxígeno molecular, al menos en la cantidad que ahora observamos en la atmósfera. Se estima que la tierra se formó de polvo estelar hace cerca de 4500 millones de años, y las evidencias más antiguas de vida corresponden a 4000 millones de años, lo que significa que por un periodo de 500 millones de años la tierra fue como cualquier otro planeta conocido, sin vida. Las primeras formas de vida que aparecieron en nuestro planeta semejaban mucho a las bacterias actuales, microscópicas y unicelulares. Estos organismos poblaron todos los nichos ecológicos existentes en aquel momento, y su metabolismo, es decir, las reacciones químicas 
que ocurren dentro de las células para obtener energía y material para construir otras células, dependía de oxidar los nutrientes con aceptores de electrones como sulfatos $\left(\mathrm{SO}_{4}{ }^{2-}\right)$, nitratos $\left(\mathrm{NO}^{3-}\right)$, o sulfuros $\left(\mathrm{S}^{2-}\right)$. Como estos aceptores finales de electrones son menos ávidos de electrones que el oxígeno molecular, la energía obtenida de la oxidación es menor a la obtenida por respiración usando oxígeno molecular.

Las primeras evidencias de fotosíntesis oxigénica aparecieron hace unos 3000 millones de años, lo que implica que por casi mil millones de años la vida fue anaerobia. Si bien, eventualmente, la selección natural favoreció a los microorganismos capaces de obtener energía a partir de una fuente luminosa (radiación solar), mediante fotosíntesis y eventualmente a los fotosintéticos oxigénicos, la concentración de oxígeno molecular en la atmósfera no aumentó inmediatamente.

Conforme aparecía, por su reactividad, oxidó compuestos o elementos que se encontraban en la tierra, principalmente hierro, precipitándolo del agua marina y formando los depósitos enormes de óxido ferroso $\left(\mathrm{FeO}_{2}\right)$ que se encuentran en los fondos marinos y que gracias a movimientos geológicos podemos observar en la corteza terrestre. Pasaron casi mil millones de años antes que el oxígeno alcanzara una concentración adecuada para que otros microorganismos lo pudieran utilizar como aceptor final de electrones. Se estima que la concentración de oxígeno molecular en la atmósfera debió haber sido al menos $15 \%$. En la actualidad la concentración es de $21 \%$, siendo el segundo gas más abundante en la atmósfera después del nitrógeno que es de $70 \%$.

\section{La gran oxidación; vida}

El aumento de oxígeno en la atmosfera fue un suceso extraordinario para la vida en el planeta, tanto así que al fenómeno se le ha llamado "El evento de la gran oxidación" que ocurrió hace unos 2000 millones de años. La elevación de la concentración de oxígeno en la atmósfera tuvo consecncias para una parte las formas vivientes existentes en ese entonces. Para los organismos anaerobios resultó fatal; para estos organismos este gas es venenoso, ya que no poseen mecanismos para evitar ser oxidados. Los organismos aerobios adquirimos, progresvamente y por presiones de selección, diversos mecanismos físicos, químicos y enzimáticos no solo para tolerar un ambiente oxidante, sino hasta para usarlo.

Se ha hipotetizado que el aumento en la concentración de oxígeno en la atmósfera ha causado varias extinciones masivas, pero no hay evidencia sólida que permita apoyar estas hipótesis. El aumento en la concentración de oxígeno molecular en la atmósfera ha sido, no solo gradual, sino en pulsos, cada uno provocado por condiciones abióticas ${ }^{5}$, como la tectónica de placas y las glaciaciones. Evidencias geológicas y paleontológicas indican que cada aumento significativo de concentración de oxígeno molecular atmosférico ha causado una radiación prolífica de especies. Uno de estos aumentos en concentración provocó la

${ }^{5}$ Abiótico: no dependiente de seres vivos. 
aparición de los eucariotas, células complejas con núcleo, los organismos unicelulares ancestros de los seres multicelulares, que incluyen plantas, hongos y animales. Un evento adicional de aumento de la concentración de oxígeno molecular dio lugar a una proliferación de especies multicelulares al principio del periodo Cámbrico, que empezó hace unos 500 millones de años.

Así como la aparición de plantas e insectos gigantes durante el periodo Carbonífero y Pérmico temprano, hace unos 300 millones de años; quizá también causó la aparición de los dinosaurios. En esa época, la concentración de oxígeno en la atmósfera alcanzó el 30\%.

Se sabe la concentración de los gases de la atmósfera en periodos ancestrales por dos medios: los gases que forman la atmósfera en algún momento quedan atrapados en burbujas dentro del hielo en los glaciares y en rocas que en ese momento se formaron. Estos gases son analizados y cuantificados por métodos físicos, lo que además permite saber la temperatura del aire en esa época.
Por el contrario, periodos de extinción masiva han ocurrido cuando la concentración de oxígeno molecular ha bajado.

..es fácil concluir que el oxígeno molecular, su aparición y aumento en la concentración en la atmósfera ha permitido una mayor diversidad de especies..

Laasíllamada metafóricamente "Madre de todas las extinciones" que ocurrió durante la época tardía del Pérmico, hace 250 millones de años. Por lo anterior, es fácil concluir que el oxígeno molecular, su aparición y aumento en la concentración en la atmósfera ha permitido una mayor diversidad de especies, sobre todo de aquellas formadas por células complejas, que contienen organelos, como núcleo y mitocondrias y multicelulares, como plantas y animales, y que su ausencia ha causado extinción.

No es exagerado decir que el oxígeno molecular es bueno, metafóricamente, para la vida.

\section{Muerte}

Debido a que el oxígeno es electronegativo, y por ende ávido de electrones, tiende a oxidar todo átomo o compuesto con un potencial redox positivo. Cuando los primeros fotosintetizadores oxigénicos empezaron a liberar el oxígeno molecular debieron, a la par, adquirir mecanismos que evitaran que el oxígeno molecular oxidara sus componentes químicos. Los organismos aerobios también evolucionaron mecanismos de protección contra el oxígeno molecular. Si bien, este cambió amplió la diversidad biológica, el trayecto evolutivo que permitió que los organismos pudieran evitar el daño provocado por el oxígeno tomo varios millones de años, a través de los cuales las especies que no poseían estos mecanismos de "defensa antioxidante" se extinguieron y las especies que adquirieron la capacidad de lidiar con el oxígeno molecular sobrevivieron. Otras especies incluso evolucionaron estrategias para tolerar el oxígeno y usarlo como aceptor final de electrones, lo que les dio una enorme ventaja evolutiva: obtener más energía al oxidar nutrientes.

Estos son los organismos aerobios. Así, las especies, que 
adquirieron mecanismos para usar el oxígeno son los ancestros de las especies actuales. Otros que no adquirieron el fenotipo para lidiar con el oxígeno molecular se les conoce como anaerobios y viven en sitios en que la concentración de oxígeno molecular es muy baja, como en los intestinos. Hay algunas especies que adquirieron la capacidad de respirar si hay oxígeno molecular o fermentar en su ausencia y se les llama los fermentadores facultativos, tales como la levadura que produce alcohol en cerveza o vino.

Aquí ya esbozamos la
capacidad del oxígeno de
causar daño por oxidación de componentes celulares. De esta propiedad se deriva una rama de la ciencia que estudia el efecto del oxígeno en aspectos como salud y envejecimiento, temas de importancia para el humano; el envejecimiento es la principal causa de sufrimiento, incapacidad, enfermedad $y$ muerte en el humano. La probabilidad de morir entre los 30 y 70 años de edad aumenta 30\%. Es paradójico que, a pesar del desarrollo de disciplinas de la ciencia que han prometido mejoras a la vida del humano, como la genética, genómica, biología molecular y los proponentes del radical libre, los factores que afectan la expectativa de vida no son, todavía, conocidos.

Existen varias hipótesis para explicar por qué envejecemos. Estas hipótesis las podemos agrupar en las que postulan "un daño celular acumulable" y las "basadas en programa." Las primeras explican el envejecimiento por daño acumulado a las células del cuerpo durante la vida, proponen que el daño es un subproducto de procesos celulares normales o consecuencia de un sistema ineficiente de reparación en las células; está relacionado con la interacción con el medio ambiente. Los proponentes del envejecimiento programado argumentan que es el resultado, no del azar, sino de un proceso regulado desde el genoma ${ }^{6}$.

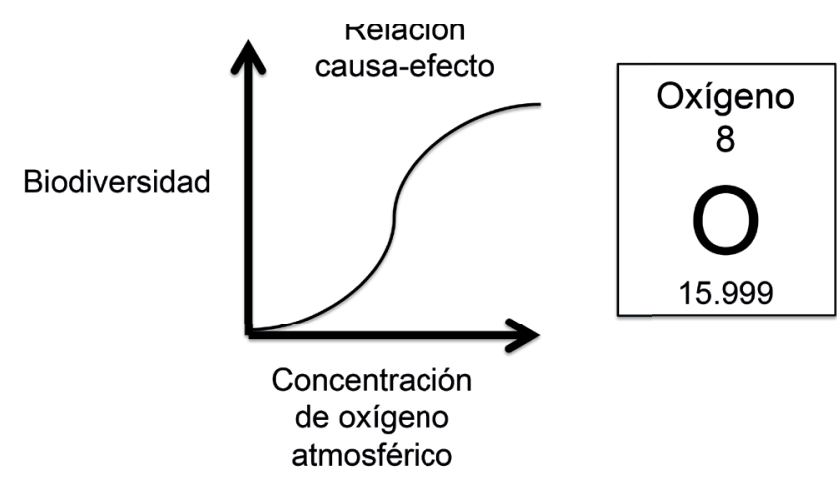
Figura 3. "Oxígeno, dador de vida" El aumento de oxígeno en la atmósfera causó la
enorme biodiversidad actual

Aquí presentamos una lista de hipótesis sobre las posibles causas del envejecimiento. Hipótesis (aquí en plural) de: longevidad programada, endócrina, inmunológica, desgaste celular, velocidad de vida, de entrecruzamiento, daño somático al DNA, telómeros, y radical libre.

La hipótesis del radical libre se menciona al final ya que tiene que ver con el tema de este ensayo: el oxígeno. Como ya se ha mencionado, el oxígeno molecular es usado como aceptor final de electrones en una ruta metabólica ${ }^{7}$ que ocurre en la mitocondria, el organelo subcelular responsable de la obtención de la mayoría de la energía en la célula.

${ }^{6}$ Conjunto de genes y su disposición de un organismo.

${ }^{7}$ Metabolismo: Conjunto de reacciones químicas que ocurren en la célula 
Sin entrar en detalles bioquímicos y de biología celular, es suficiente mencionar que, en la mitocondria, los electrones tomados de nutrientes son transportados por una serie de moléculas organizadas secuencialmente, conocida como "La cadena respiratoria," al final de la cual el oxígeno toma esos electrones para formar agua. La reducción completa de oxígeno molecular requiere de cuatro electrones. La cadena respiratoria no es $100 \%$ eficiente, y continuamente se generan derivados parcialmente reducidos de oxígeno, conocidos como especies reactivas de oxígeno o ROS. Esta característica hace que sean más ávidos por electrones, aumentando su reactividad. Los ROS reaccionan con cualquier molécula con potencial redox positivo, ya sea ADN, lípidos o proteínas.

Son varios los derivados de oxígeno parcialmente reducido producidos por este mecanismo.

El ión superóxido $\left(\mathrm{O}_{2}^{-}\right)$,peróxido $\left(\mathrm{H}_{2} \mathrm{O}_{2}\right)$, y el radical hidroxilo $(\mathrm{OH} \cdot)$, todos ellos capaces de provocar mutaciones en los genes, de ahí su toxicidad. Como ya se mencionó, las especies han adquirido, por medio de evolución, mecanismos para deshacerse de los efectos adversos de estos potenciales agresores de las células. Entre ellos hay dos principales: 1) la síntesis de moléculas con un potencial redox más positivo que las moléculas a proteger, por lo que reaccionan neutralizando a los ROS; se les llama agentes antioxidantes por que evitan la oxidación de moléculas vitales para la célula. Este mecanismo consume una molécula de antioxidante por cada derivado de oxígeno parcialmente reducido, ¡es suicida! Y 2) el otro mecanismo es enzimático. Las enzimas son moléculas con la capacidad de facilitar reacciones químicas.

Entre otras, las de eliminación de derivados de oxígeno parcialmente reducido.

Tres grupos principales de enzimas están involucradas. Las superóxido dismutasas, las catalasas y las peroxidasas. Como cada molécula de enzima puede llevar a cabo la transformación de miles de moléculas de sustrato, este mecanismo es más eficiente. Cada mecanismo tiene sus ventajas y limitaciones.
Para tratar de ayudar a las células a lidiar con los ROS se ha generado una industria de producción masiva de "agentes antioxidantes". Hoy podemos encontrar una enorme variedad de productos que ofrecen el beneficio de mejorar la salud y evitar envejecimiento, productos que sólo han servido para enriquecer a los industriales. Incluso un premio Nobel, el Dr. Linus Pauling, cuando retirado, se dedicó a promover el consumo masivo de vitamina C, un antioxidante. Él sugería tomar un gramo diario, y 10 gramos diarios para reducir la incidencia de cáncer; el doble ganador de premios Nobel, Química y La Paz, murió en descrédito de la comunidad científica por su iniciativa, por no estar sustentada en investigación científica. Otro reclamo no fundamentado se da por medio de anécdotas, el consumo de "alimentos antioxidantes". Estos como toda moda basada en anécdotas cambia regularmente, ya pasó la moda de la guanábana y ahora se promueve la Stevia.

Por otro lado, la producción de ROS es parte del metabolismo 
natural de las células. Estos derivados de oxígeno parcialmente reducido son usados por algunas células blancas de la sangre para oxidar componentes de agentes infecciosos y eliminarlos.

Finalmente, como todo fenómeno en la naturaleza, el envejecimiento debe ser multifactorial, es decir, depende de varios factores y más importante, es posible que no sólo sean los factores mencionados los que la causen, sino la forma como ellos interactúan. La hipótesis del radical libre del envejecimiento no ha podido explicar por qué la expectativa de vida del humano pasó de 40 a 70 años en el siglo XX y por qué los atletas que consumen más oxígeno viven más, gracias a que los radicales libres estimulan una respuesta adaptativa conocida como hormesis.

¡Carpe diem!

\section{Agradecimientos}

Los Autores agradecemos al Lic. Gerardo Hernández el diseño gráfico editorial y a la Ms.C. Diana Dorantes la revisión del Idioma Inglés del Abstract.

\section{Literatura sugerida}

Cuatro libros de Nike Lane, University College London, son altamente recomendados

Lane N. 2004. Oxygen: the molecule that made the world. Oxford University Press. 384 pages

Lane N. 2006. Power sex and suicide: mitocondria and the meaning of life. Oxford University Press. 368 pages.

Lane N. 2009. Life ascending: the ten great inventions of evolution. W.W. Norton \& Co. 352 pages.

Lane N. 2015. The Vital Question: Energy, Evolution, and the Origins of Complex Life.W.W. Norton \& Co. 368 pages.

Harman D. 2009. Origin and evolution of the free radical theory of aging: a brief personal history, 1954-2009. Biogerontology 10:773-781.

Fridovich I. 1997. Superoxide Anion Radical (O2 -), Superoxide Dismutases, and Related Matters. Journal Biological Chemistry 272:1851518517.

Jin K. 2010. Modern Biological Theories of Aging. Aging Diseases 1:72-74.

Offord C. 2016. Mitochondrial activity predicts fish life span? The scientist. (http://www.the-scientist.com/?articles.view/ articleNo/45441/title/Mitochondrial-Activity-Predicts-FishLife-Span-/\&utm_campaign=NEWSLETTER_TS_The-ScientistDaily_2016\&utm_source=hs_email\&utm_medium=email\&utm_ content $=26636573$ \&_hsenc $=$ 2ANqtz-8SifcZXJ2nEGQkkm7aC4jZx 7Ts6Q1oDAgJL52Ek3cLNqGk0Ad7PEROPGu91uDn4drUfR8z076P 09L7qaAlycXyq1nANQ\&_hsmi=26636573/) 
Cita de este artículo

García-Carreño F., A. Sánchez Paz, M. A. Navarrete del Toro. 2016. La oxidación, dualidad vida y muerte. Recursos Naturales y Sociedad, Vol. 2 (2): 12-23. https://doi.org/10.18846/renaysoc.2016.02.02.02.0001

Sometido: 21 de Mayo de 2016

Revisado: 22 de Agosto de 2016

Aceptado: 13 de Septiembre de 2016

Editora asociada: Dra. Norma Hernández Saavedra

Idioma Inglés Abstract: Ms.C. Diana Dorantes

Diseño gráfico editorial: Lic. Gerardo Hernández 